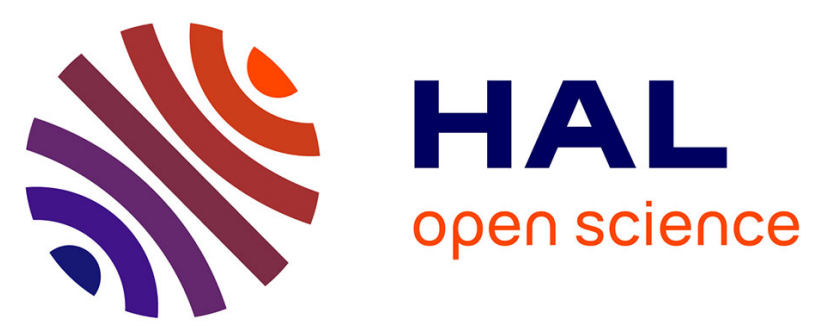

\title{
Webstrates: demonstrating the potential of Shareable Dynamic Media
}

Clemens N Klokmose, James N Eagan, Siemen Baader, Wendy Mackay, Michel N Beaudouin-Lafon

\section{To cite this version:}

Clemens N Klokmose, James N Eagan, Siemen Baader, Wendy Mackay, Michel N Beaudouin-Lafon. Webstrates: demonstrating the potential of Shareable Dynamic Media. 19th ACM conference on Computer-Supported Cooperative Work and Social Computing, ACM, Feb 2016, San Francisco, CA, United States. pp.61 - 64, 10.1145/2818052.2874325 . hal-01435919

\section{HAL Id: hal-01435919 https://hal.science/hal-01435919}

Submitted on 19 Jan 2017

HAL is a multi-disciplinary open access archive for the deposit and dissemination of scientific research documents, whether they are published or not. The documents may come from teaching and research institutions in France or abroad, or from public or private research centers.
L'archive ouverte pluridisciplinaire HAL, est destinée au dépôt et à la diffusion de documents scientifiques de niveau recherche, publiés ou non, émanant des établissements d'enseignement et de recherche français ou étrangers, des laboratoires publics ou privés. 


\section{Webstrates: demonstrating the potential of Shareable Dynamic Media}

\author{
Clemens N. Klokmose \\ Aarhus University \\ Aarhus, Denmark \\ clemens@cs.au.dk
}

James Eagan

Télécom ParisTech, CNRS

Paris, France

james.eagan@telecom-

paristech.fr

Siemen Baader

Aarhus University

Aarhus, Denmark

sb@cs.au.dk

\author{
Wendy Mackay \\ Inria, Univ. Paris-Sud, CNRS, \\ Université Paris-Saclay \\ Orsay, France \\ mackay@Iri.fr
}

Michel Beaudouin-Lafon

Univ. Paris-Sud, CNRS, Inria,

Université Paris-Saclay

Orsay, France

mbl@|ri.fr

Clemens N. Klokmose, James Eagan, Siemen Baader, Wendy Mackay, Michel Beaudouin-Lafon. Webstrates: demonstrating the potential of Shareable Dynamic Media. In Companion Proceedings of the 19th ACM conference on

Computer-Supported Cooperative Work and Social Computing (CSCW 2016), ACM, February 2016. pp 61-64.

(c) is held by the owner/author(s)

CSCW' 16 Companion, February 27-March 02, 2016, San Francisco, CA, USA ISBN 978-1-4503-3950-6/16/02.

http://dx.doi.org/10.1145/2818052.2874325

\begin{abstract}
Shareable Dynamic Media is our vision for an interactive computing environment that blurs the distinction between documents and applications, characterized by three key properties: malleability, so that users can appropriate and repurpose media in idiosyncratic ways; shareability, so that users can collaborate on any type of content; and distributability, so that users can access and manipulate media across diverse devices and platforms. Webstrates is a prototype web-based environment designed to explore this vision. It supports real-time sharing of any web content, as well as transclusion to flexibly combine and assemble multiple media together. The demonstration illustrates the power of Webstrates and Shared Dynamic Media through several scenarios, such as collaborative authoring, distributed slideshow presentation with audience participation, collaborative programming, and shareable window management.
\end{abstract}

\section{Author Keywords}

Real-time Collaborative Documents; Dynamic Media; Web.

\section{ACM Classification Keywords}

H.5.2 [User Interfaces]

\section{Introduction}

Alan Kay's early vision of interactive computing saw software as an expressive medium that could be personalized, 
tinkered with and shared. He called it Personal Dynamic Media [2]. For example, children with linked Dynabooks could collaboratively tinker with a Spacewar game to make it more challenging by adding a more sophisticated form of gravity [3]. Decades later, Mark Weiser envisioned a future of ubiquitous computing [6], where heterogenous devices of varying sizes and capabilities interact easily with each other and technology disappears into the background. $\mathrm{He}$ imagined how colleagues would share a virtual office and collaborate on a document, seamlessly moving between a wall-sized display and various "tabs" and "pads".

Today's interactive software traps documents inside closed applications, making it difficult to truly share content, personalize applications, and work across devices. While the hardware envisioned by both Kay and Weiser has by and large been realized today, software lacks behind.

Our vision, Shareable Dynamic Media, builds on Kay and Weiser's visions. We define shareable dynamic media as collections of information substrates (or substrates for short). Substrates are software artifacts that embody content, computation and interaction, effectively blurring the distinction between documents and applications.

Substrates can evolve over time and shift roles, acting as what are traditionally considered documents in one context and applications in another, or a mix of the two. For example, a document can contain a set of tools to edit other documents, and those tools can themselves be edited as a regular document. Or a document can contain regular content, e.g. text, as well as tools to edit it. Substrates may be composed in various ways, e.g., one substrate can give meaning and structure to another. For example, a bar-chart substrate can define how to visualize a statistical data substrate.
Shareable Dynamic Media embodies three key properties:

- Malleability: users can appropriate their tools and documents in personal and idiosyncratic ways:

- Shareability: users can collaborate seamlessly on multiple types of data within a document, using their own personalized views and tools; and

- Distributability: tools and documents can move easily across different devices and platforms, and multiple devices can be combined to provide a consistent environment.

\section{Webstrates}

Webstrates [4] (web substrates) is a prototype of shareable dynamic media that consists of a custom web server that serves pages, called webstrates, to regular web browsers. Each webstrate is a shared collaborative object: changes to the webstrate's Document Object Model (DOM), as well as changes to its embedded JavaScript code and CSS styles, are transparently made persistent on the server and synchronized with all clients sharing that webstrate, using Operational Transformations [1].

By sharing the DOM itself, rather than some data model from which is derived the content of a web page, as most current web frameworks do, Webstrates supports a strong form of sharing that is easy to understand. Any change to the DOM, whether it is made directly by the user or through embedded code, is visible by everyone.

By also sharing embedded code, behavior typically associated with application software can also be (collaboratively) manipulated, opening the way to novel possibilities for combining content, computation and interaction. Sharing a webstrate that contains, e.g., a button, also shares the code of 

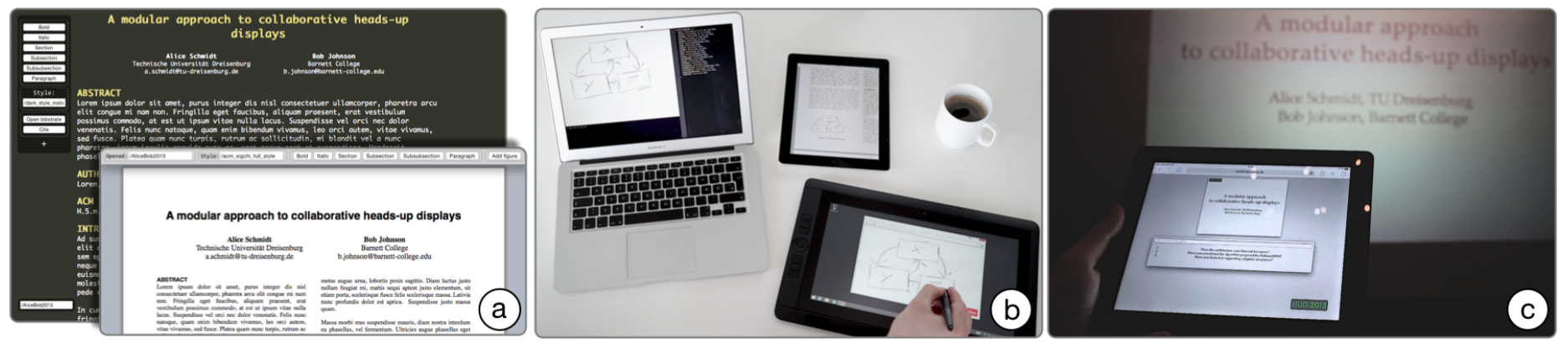

Figure 1: Sample uses of Webstrates: (a) Collaborative document authoring with different editors personalized at run-time; (b) Multiple devices used to sketch a figure (tablet 1), see it in a print preview (tablet 2), and adjust it in a graphics editor (laptop). (c) Distributed talk controlled remotely by a speaker with a separate interface for audience participation.

that button, which can be modified by any user of the webstrate.

The true power of webstrates comes from the ability to compose them by embedding one webstrate within another a process called transclusion [5]. Transclusion lets users truly share, rather than copy, content. For example, a webstrate containing editing tools can transclude a webstrate of the document being edited, creating something similar to a traditional application. Another user can use a different editor webstrate, with a different set of tools, and transclude the same content webstrate. This results in a shared editor where each user can choose their editing tools.

\section{Background}

Webstrates is described in a paper [4] to appear at the 28th annual ACM Symposium on User Interface Software and Technology (UIST '15) in November 2015, nominated for the best paper award. The Webstrates platform is opensource and available for download at www.webstrates.net.
The UIST 2015 article describes how Webstrates can be used to allow users to collaborate on the same documents with their own personalized and extensible editors (Figure 1a). It shows that webstrates enable collaborative runtime extension of a user interface, e.g., one user can manipulate the user interface of another remotely and at runtime. It also shows how webstrates make it possible to simultaneously sketch a figure on a graphics tablet, see it in a live print preview embedded in a paper shown on an iPad, and adjust it in a vector graphics editor on a laptop (Figure 1b). Finally it demonstrates how webstrates can be used to easily orchestrate a complex setup for a distributed slideshow presentation that includes audience participation (Figure 1c).

The entire authoring of the UIST 2015 paper [4], including the production of the camera-ready PDF file, was done by the five authors, living in four different locations, using Webstrates. The development of Webstrates was also done collaboratively in part using Webstrates. 


\section{Relevance for the CSCW audience}

Collaboration is at the core of our vision of shareable dynamic media. We advocate for a software paradigm where support for collaboration is the norm rather than the exception, i.e. where real-time sharing is built into the system rather than under the responsibility of the application.

Webstrates is a prototype that demonstrates a practical realization of this vision, and a platform enabling the development of advanced collaborative systems. The scenarios in the demonstration all emphasize the collaborative aspect of Webstrates.

The implementation of Webstrates uses Operational Transformation through the ShareJS Javascript library (http:// sharejs.org). It illustrates a practical and original use of a long line of research published at CSCW on consistency maintenance or replicated state.

\section{Description of the demonstration}

The demo shows a series of applications built with Webstrates ranging from collaborative paper writing, distributed slideshow presentation, collaborative programming to shareable window managers

The collaborative paper writing demonstration is inspired by our use of Webstrates to author the UIST 2015 paper. It illustrates how multiple authors can share the document being edited but use different visualisations of the paper as well as different editing tools, including on-the-fly creation of new tools and live editing of another user's editor.

The distributed slideshow demonstration is inspired by the first author's use of Webstrates to give a number of talks. The audience view is shown on the shared screen while the presenter view, shown on a tablet, share the slides but provides the presenter with additional tools as well as the presenter notes. The audience also has access to a view where they can ask questions, which show up on the last slide of the presentation. This demonstration illustrates the use of transclusion for the complex orchestration of different views.

The collaborative development demonstration shows how Webstrates can be used for collaborative editing of the code of the webstrates themselves. It features a shared code editor and demonstrates the live effects of changing the CSS style sheet of a webstrate, or the Javascript code of a tool embedded into an editor.

The shareable window manager demonstration shows how webstrates can recreate a traditional window manager, with the unique capability of sharing windows and their content.

\section{References}

[1] Clarence A Ellis and Simon J Gibbs. 1989. Concurrency control in groupware systems. In Acm Sigmod Record, Vol. 18. ACM, 399-407.

[2] Alan Kay and Adina Goldberg. 1977. Personal dynamic media. Computer 10, 3 (1977), 31-41.

[3] Alan C Kay. 1972. A personal computer for children of all ages. In Proceedings of the ACM annual conference-Volume 1. ACM, 1.

[4] Clemens N. Klokmose, James Eagan, Siemen Baader, Wendy Mackay, and Michel Beaudouin-Lafon. 2015. Webstrates: Shareable Dynamic Media. In Proceedings of the 28th annual ACM symposium on User interface software and technology (UIST '15). ACM.

[5] Theodor H Nelson. 1995. The heart of connection: hypermedia unified by transclusion. CACM 38 (1995), 31-33.

[6] Mark Weiser. 1991. The computer for the 21st century. Scientific american 265, 3 (1991), 94-104. 